\title{
Analekzem
}

\section{Unverzichtbare Steroide}

_ Perianaler Juckreiz wird meist durch Stuhlsekret verursacht, das ein irritativtoxisches Ekzem auslöst. Flohsamenschalen reduzieren die Episoden von Stuhlschmieren um $80 \%$. In der Akuttherapie haben darüber hinaus Steroidsalben und -cremes einen festen Platz. Beim akuten Analekzem rät der Dr. Bernhard Lenhard, Heidelberg, zur Therapie mit 1\%igem Hydrocortison in Basisemulsion. Nicht vernachlässigt werden darf die Therapie der proktologischen Grunderkrankungen mit dem sekretierenden Hamörrhoidalleiden als einen derHauptauslöser.

Beim häufigen irritativ-toxischen Analekzem empfiehlt Lenhard initial
Steroidlotionen oder -cremes mit einem mittelstarken Kortikosteroid, gefolgt von Hydrocortison-Präparaten (z.B. Postericort ${ }^{\oplus}$ ). Diese haben ein geringeres Atrophierisiko und können auch etwas länger eingesetzt werden. Sie eignen sich zudem für die Intervalltherapie bei chronisch-rezidivierenden Formen. Bei akutem Analekzem ist placebokontrolliert-doppelblind nach 7-9 Tagen eine signifikante Reduktion von Juckreiz und Brennen bei gleichzeitiger guter Verträglichkeit dokumentiert.

Dr. Renate Leinmüller

- Symposium „Proktologie für die Praxis", 43. Deutscher Koloproktologen-Kongress; München, März 2017 (Veranstalter: Dr. Kade)

\section{Impfstoffausschreibungen abgeschafft}

Das Arzneimittel-Versorgungsstärkungsgesetz hat die entsprechende Regelung ( $§$ 132e Abs. 2 SGB V) aufgehoben. Die Impfstoffausschreibungen durch gesetzliche Krankenkassen hatte dazu geführt, dass die Vergabekriterien ausschließlich wirtschaftlichen Aspekten folgten und weniger der bestmöglichen Versorgung der gesetzlich versicherten Patienten in Deutschland dienten. Diesen Versicherten werden derzeit z. B. Impfstoffe mit einem breiteren Schutz versagt - etwa bei der saisonalen Grippeimpfung, wogegen privat versicherte Patienten davon profitieren können. Seit Inkrafttreten des Gesetzes zum 1. April 2017 können nunmehr die Impfstoffe aller Hersteller zu Lasten der GKV verordnet werden.

Red.

- Nach Informationen von GSK

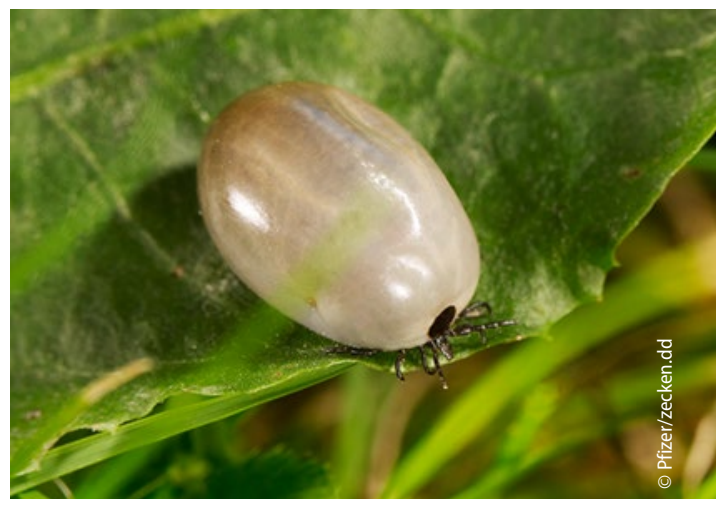

Mit Blut vollgesaugte Zecke auf einem Blatt.

rologischer Defizite wie Lähmungen, Gleichgewichtsstörungen, Kopfschmerzen, Fatigue oder kognitive Störungen etc. nach überstandener FSME-Erkrankung.

Den zuverlässigsten Schutz vor einer FSME-Erkrankung bietet die Impfung. Die Ständige Impfkommission (STIKO) empfiehlt sie allen Personen, die sich in FSME-Risikogebieten aufhalten und dort in Beruf oder Freizeit zeckenexponiert sind. Wie wirksam die Impfprophylaxe tatsächlich sein kann, zeigt sich in Österreichs: Dort wurde eine Durchimpfungsrate von rund $90 \%$ erreicht. In der Folge sank die Anzahl gemeldeter FSME-Infektionen auf wenige Fälle pro Jahr.

Dr. Brigitta Schneider

- Pressegespräch „FSME - die unterschätzte Gefahr: höhere FSME-Zahlen 2016 versus stagnierende Impfraten"; München, März 2017 (Veranstalter: Pfizer) 